\title{
Cross-modal psychological refractory period in vision, audition, and haptics
}

\author{
Pei-Luen Patrick Rau ${ }^{1}$. Jian Zheng ${ }^{1}$ \\ Published online: 12 February 2020 \\ (C) The Psychonomic Society, Inc. 2020
}

\begin{abstract}
People's parallel-processing ability is limited, as demonstrated by the psychological refractory period (PRP) effect: The reaction time to the second stimulus (RT2) increases as the stimulus onset asynchrony (SOA) between two stimuli decreases. Most theoretical models of PRP are independent of modalities. Previous research on PRP mainly focused on vision and audition as input modalities; tactile stimuli have not been fully explored. Research using other paradigms and involving tactile stimuli, however, found that dual-task performance depended on input modalities. This study explored PRP with all the combinations of input modalities. Thirty participants judged the magnitude (small or large) of two stimuli presented in different modalities with an SOA of 75-1,200 ms. PRP effect was observed, i.e., RT2 increased with a decreasing SOA, in all the modalities. Only in the auditory-tactile condition did the accuracy of Task 2 decrease with a decreasing SOA. In the auditory-tactile and tactile-visual conditions, RT to the first stimulus also increased with a decreasing SOA. Current models could only explain part of the results, and modality characteristics help to explain the overall data pattern better. Limitations and directions for future studies regarding reaction time, task difficulty, and response modalities are discussed.
\end{abstract}

Keywords Psychological refractory period $\cdot$ Central bottleneck $\cdot$ Dual-task $\cdot$ Multisensory perception $\cdot$ Multimodal display

\section{Introduction}

People often have to perform multiple tasks simultaneously. For example, drivers control their cars' speed and direction, while monitoring other cars and passengers nearby and sometimes heeding their phones. Moreover, some information is received by hearing and touch, such as horn sounds and vibrations caused by speed bumps. The human's ability to multitask, however, has a capacity limitation, which constrains performance and may lead to serious consequences such as accidents.

One of the main paradigms for studying multitasking is the psychological refractory period (PRP). It indicates that when two stimuli (S1 and S2) are presented with short stimuli onset asynchrony (SOA) and both require fast responses (R1 and

Pei-Luen Patrick Rau

rpl@tsinghua.edu.cn

1 Institute of Human Factors and Ergonomics, Department of Industrial Engineering, Tsinghua University, South 525, Shunde Building, Beijing 100084, China
R2), the reaction times to the second stimulus (RT2) increases with decreasing SOA (Pashler, 1994).

\section{Theories of the psychological refractory period (PRP)}

The dominant account of the PRP effect is the responseselection bottleneck (RSB) model (Pashler, 1994; Welford, 1952). It generally divides the processing of a task into three phases: the perceptual processing, the response selection, and the response execution; response selections for two tasks cannot be carried out simultaneously. According to the RSB model, the response time to the first target (RT1) does not depend on SOA, and RT2 decreases with a slope of -1 if plotted against SOA when SOA is shorter than RT1. Many studies have supported the RSB model (e.g., Ruthruff et al. 2009; Ruthruff et al. 2003). Recently, it has been argued that the central bottleneck is not located in response selection but in the timing of response initiation (Klapp, Maslovat, \& Jagacinski, 2019). Some PRP studies have found a backward crosstalk effect: the performance of Task 1 was better if R2 was congruent with R1 (Hommel, 1998; Hommel \& Eglau, 2002). The RSB model could not explain the backward crosstalk effect, and some updated versions of RSB have been 
proposed (Hommel, 1998; Janczyk, Renas, \& Durst, 2018) to account for this effect. Klapp et al.'s (2019) model also allows a backward crosstalk effect to exist.

The central capacity-sharing model (Tombu \& Jolicoeur, 2003) introduced an additional parameter: the sharing portion of central capacity to Task 1 . The central capacity-sharing model assumes there is no bottleneck in processing, and processing to two tasks could be carried out at the same time. The RPR effect is caused by a limited central capacity. Although the central capacity-sharing model does not assume any bottleneck, it includes the RSB model as a special case where the sharing portion equals one. When the portion is less than one, the central capacity is shared between the two tasks. According to the central capacity-sharing model, RT2 decreases with a slope of -1 if plotted against SOA when SOA is shorter than RT1; when the sharing portion is less than one, RT1 decreases as SOA increases, and the backward crosstalk effect is possible. Studies about the backward crosstalk effect support the central capacity-sharing model over the RSB model (e.g., Huestegge \& Koch, 2010; Stephan \& Koch 2010). Besides, in most $(67.1 \%)$ of the studies that have analyzed the effect of SOA on RT1, RT1 was not independent of SOA (Strobach et al. 2015).

The executive control of the theory of visual attention (Logan \& Gordon, 2001) combines the theory of visual attention (Bundesen, 1990) and the exemplar-based random walk model (Nosofsky \& Palmeri, 1997). It explains the PRP effect by running the theory of visual attention twice. Executive control of the theory of visual attention assumes that both tasks have access, but with different and dynamic priorities, to the central capacity under short SOA, and that the earlier processing of Task 2 is largely inhibited (e.g., to 10\%) after the response selection of Task 1 . According to the executive control of the theory of visual attention, RT1 decreases as SOA increases, the backward crosstalk effect should be observed, and RT2 decreases with a slope even less than -1 if plotted against SOA when SOA is shorter than RT1. Figure 1 presents the comparison of three models.

\section{Effect of modality}

All the models mentioned above are independent of (input and output) modalities. However, some recent studies supported the notion that dual-task performance depended on modalities (Huestegge \& Hazeltine, 2011; Morrison, Burnham, \& Morrison, 2015). Dual-task costs are affected by ideomotor compatibility, i.e., the similarity between the stimuli and the feedback of responses (Greenwald, 1972; Greenwald \& Shulman, 1973; Lien, McCann, Ruthruff, \& Proctor, 2005; Lien, Proctor, \& Allen, 2002). The inputoutput modality pairing also affects dual-task performance (Hazeltine \& Ruthruff, 2006; Hazeltine, Ruthruff, \& Remington, 2006): visual-manual and auditory-vocal pairings generally result in less dual-task costs than visual-vocal and auditory-manual pairings. Besides, the somatosensory system has some unique features compared

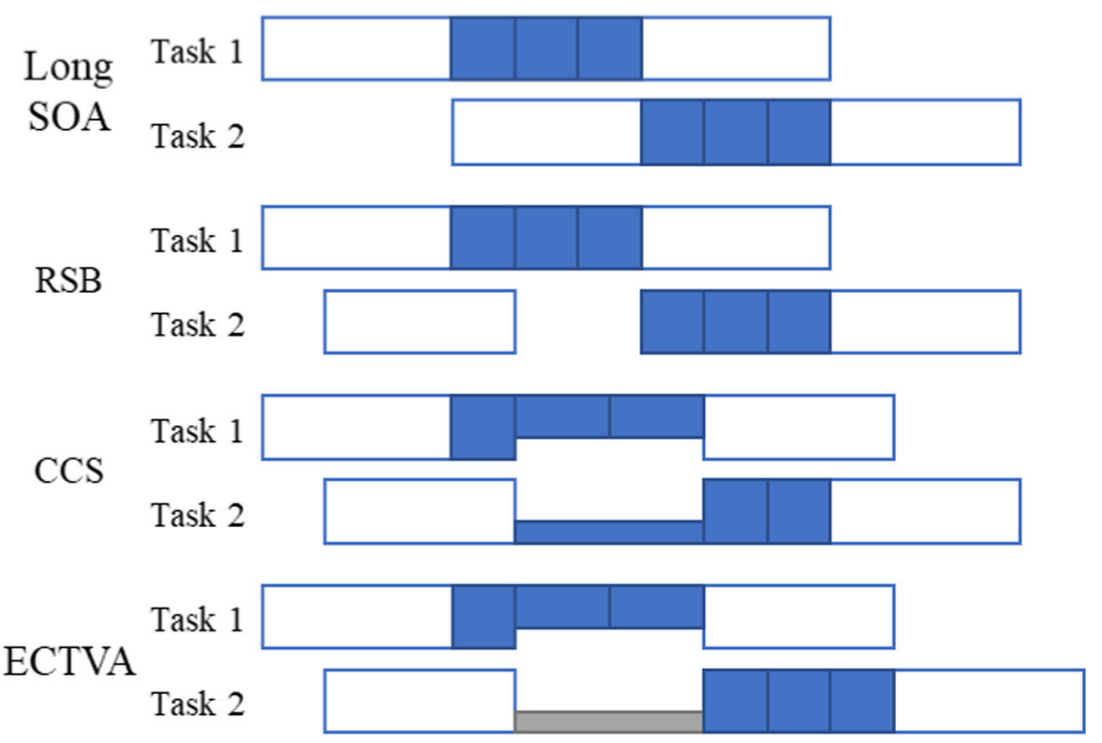

Fig. 1 The response time of two tasks predicted by response selection bottleneck (RSB), central capacity-sharing (CCS), and executive control of theory of visual attention (ECTVA) models. The response selection of Task 1 and Task 2 were assumed to take three units of central capacity to process. In CCS and ECTVA, the priority of Task 1 was assumed to be twice that of Task 2 (presented by the height of the sharing capacity), so $\mathrm{SP}$ is 0.67 . Under a long SOA, all models make the same prediction. Under a short SOA (SOA < RT1), the RSB model predicts RT1 to be

independent of SOA, and RT2 decreases with slope of -1 as SOA increases. The CCS model predicts RT1 decreases as SOA increases and the slope depends on SP, and RT2 decreases with slope of -1 as SOA increases; the ECTVA predicts RT1 decreases as SOA increases and the slope depends on the relative priority of Task 1 to Task 2, and RT2 decreases with slope less than -1 as SOA increases. The early processing of Task 2 (the gray rectangle) is largely lost after the response selection of Task 1 
with vision and audition: Somatosensory signals go through the spinal cord and processing in the brain has asymmetry in hemispheres (Coghill, Gilron, \& Iadarola, 2001; Symonds, Gordon, Bixby, \& Mande, 2006; Vallar, 2007). This evidence suggested that the role of modalities in multitasking is worth exploration (Huestegge \& Hazeltine, 2011).

However, the effect of input modalities on PRP has not been fully explored: PRP studies using tactile stimuli are very few, compared with the abundance of visual and auditory PRP studies (e.g., Dux, Ivanoff, Asplund, \& Marois, 2006; Hunt \& Kingstone, 2004; Lien et al., 2002; Pashler, 1994; Sigman \& Dehaene, 2008). Actually, Huestegge and Hazeltine (2011) reviewed 53 dual-task studies between 2006 and 2011 and found only one study involved touch. Collignon and De Volder (2009), which supposedly is the study meant, found that blind participants responded faster than those with normal sight in auditory-tactile tasks; this is irrelevant with PRP, because the SOA was over 1,500 ms. An earlier relevant study is from Brebner (1977), who found a PRP effect with tactile stimulation on both hands (as cited in Pashler, 1994).

A recent study involving visual, auditory, and tactile stimuli found that PRP existed in input modality combinations of visual-visual, auditory-visual, and tactile-visual stimuli (Hibberd, Jamson, \& Carsten, 2013): Drivers first discriminated visual, auditory, or tactile stimuli as Task 1, then, after a varying SOA, stopped their cars when the leading car's brake lights turned red. Drivers performed the braking tasks slower as the SOA decreased, and this effect was not moderated by the input modalities of Task 1. Still, the combination of audition and tactility has not been explored in previous studies on PRP. Moreover, research on crossmodal dual-task found asymmetrical influence between modalities (Arnell \& Larson, 2002; Chun \& Potter, 1995, Exp. 5; Soto-Faraco et al., 2002, Exp. 1). For instance, Arnell and Larson (2002) found that performing an auditory Task 1 affected the performance of a visual Task 2, but performing a visual Task 1 did not affect the performance of an auditory Task 1 . Therefore, although PRP has been studied with tactile-visual tasks (Hibberd et al., 2013), the combination of visual-tactile tasks still needs to be explored.

To sum up, input modality plays a key role in dual-task performance. However, few previous studies have employed tactile stimuli, and actually none incorporating audition with tactility, and none covering the visual-tactile tasks. This study will fill in these gaps by exploring and comparing the PRP effect in all the possible combinations of input modalities in one experiment. Based on previous studies, we predict the RT2 of auditory-visual, visual-auditory, and tactile-visual would decrease with a slope of -1 as SOA increases. RT2 of the other conditions, RT1, and accuracy data could not be predicted because of the lack of relevant results.

\section{Method}

\section{Participants}

Thirty participants (23 female), aged $18-30$ years $(M=23.6$, $S D=2.79$ years), took part in the experiment. All had normal or corrected-to-normal vision and normal auditory and tactile senses. They were rewarded with 36-56 CNY (equivalent to US\$5.18-8.06) according to their performance. This research was approved by the institutional review board at the Institute of Human Factors and Ergonomics, Tsinghua University. Informed consent was obtained from each participant.

We calculated the required sample size based on effect size of SOA on RT2 in a previous study (Hibberd et al., 2013) with $F$ to $\eta_{p}^{2}$ transformation (Lakens, 2013) by $\mathrm{G}^{*}$ Power (Faul, Erdfelder, Lang, \& Buchner, 2007). Because of a rather large effect size, i.e., $\eta_{p}^{2}=.55$, to achieve a power of .95 , three to 11 participants are needed. However, to be conservative and to counterbalance the order of input modalities, we still recruited 30 participants.

\section{Apparatus and stimuli}

We used an HTC vive pro to present visual stimuli in the headmounted display (HMD). Haptic stimuli were presented as vibrations on vive controllers. Auditory stimuli were presented through a pair of noise-cancelling earphones connected to the HMD. Stimuli could be of two magnitude levels: Visual stimuli were white circles with a relative area of 16:100 on a black background; auditory stimuli were rectangle sound waves with a relative volume of 16:100, and haptic stimuli were vibrations with a relative magnitude of 16:100.

\section{Design}

This study was a 6 (modality: visual-auditory, auditory-visual, visual-tactile, tactile-visual, auditory-tactile, and tactile-auditory) $\times 5$ (SOA: 75, 150, 300, 600, and 1,200 ms) withinsubject design. Modality, or the combination of input modalities, was counterbalanced between subjects. SOA was randomly selected from the five levels with equal probability for each trial. RT and accuracy data for both tasks were analyzed as advocated by Strobach et al. (2015).

\section{Task and procedure}

Information about the participants' gender and age was collected during the online recruitment. Each participant arrived at the laboratory individually, sat on a chair, put on the HMD and earphones, held one controller in each hand, and rested their elbows on the chair arms. Participants got familiar with the visual, auditory, and tactile stimuli so that they could easily 
distinguish small and large stimuli in each modality. The experiment consisted of six blocks, corresponding to the six conditions of modality, and its order was counterbalanced between subjects.

Within each block, participants completed ten training trials and then 50 testing trials. In each trial, participants first saw a fixed cross at the center of the panel. The cross disappeared after $500 \mathrm{~ms}$, and the first stimulus appeared and stayed for $100 \mathrm{~ms}$. After a random SOA, the second stimulus appeared and stayed for $100 \mathrm{~ms}$. Participants could respond to the stimuli as soon as each appeared. Trials were terminated by participants' responses to the second stimulus or after a maximum response window of $3 \mathrm{~s}$. Subsequently, a blank screen was shown during an inter-trial interval of $1 \mathrm{~s}$.

The participants' task was to judge the magnitude (small or large) of the two stimuli as accurately and as fast as possible, with the order of manual responses matching the order of stimuli occurrences. To respond, participants pointed the virtual laser from the controller to a button on the virtual panel and clicked the trigger on the controller. RT was defined as the time from stimuli appearing to the clicking action. It was very similar to using a mouse: move and click. Participants were explicitly instructed to follow these requirements: Use the left hand for Task 1 and the right hand for Task 2; do not wait until the second stimulus appears to respond to the first one; always prioritize Task 1. Figure 2 presents the interface of the experiment.

\section{Results}

We report the performance of Task 2 and then Task 1 . No trials were excluded, but using only trials with correct responses did not change the results, see Fig. 4. Greenhouse-Geisser correction was used wherever the assumptions of sphericity were violated. The data set is available at the open science framework (Rau \& Zheng, 2019).

\section{Performance of Task 2}

Table 1 presents the means and SDs of RT and accuracy of Task 2 . We conducted two 6 (modality) $\times 5$ (SOA) repeatedmeasures analysis of variance (RM-ANOVA) predicting the RT and accuracy of Task 2 separately. Table 2 presents the results. Figure 3 depicts the RT2 pattern.

The effect of SOA on RT2 was significant: RT2 decreased as the SOA increased, first with a slope of about -1 when SOA was less than $500 \mathrm{~ms}$, then with a much flatter slope. We also ran six separate RMANOVAs on the effect of SOA on RT2 within the six conditions of modality. Table 3 shows that the effects of SOA on RT2 were significant for all the conditions. Both the effect sizes and the powers were large. Therefore, PRP existed in all the six modality combinations.

Significant effects are shown in bold

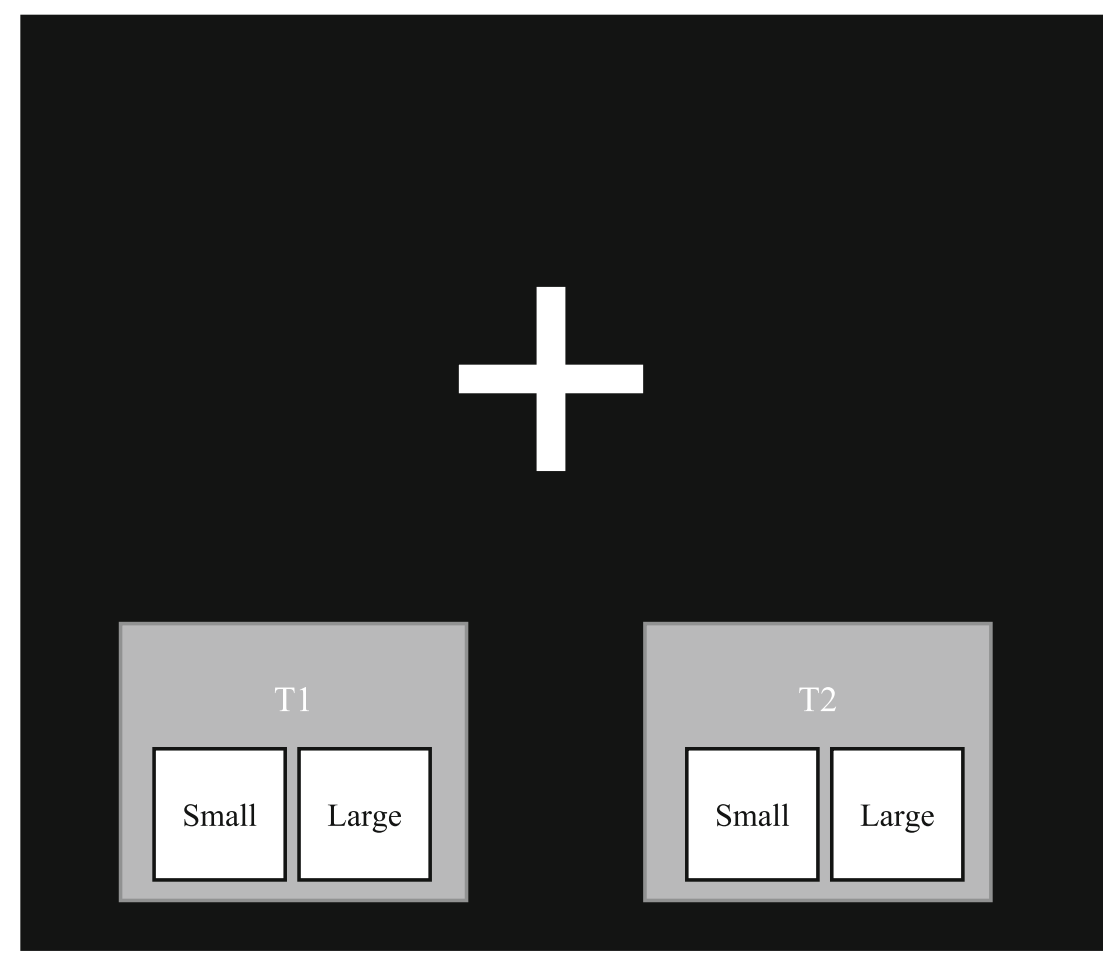

Fig. 2 The experiment interfaces. The "small" and "large" buttons were in the Chinese in experiment. T1 indicates Task 1, T2 indicates Task 2 
$R T$ reaction time, $A T$ auditory-tactile, $A V$ auditory-visual, $T A$ tactile-auditory, $T V$ tactile-visual, $V A$ visual-auditory, $V T$ visual-tactile

The main effect of modality was significant: Generally, the auditory-visual task had the longest RT2, and the tactile-visual task had the shortest RT2. Multiple comparisons with Bonferroni correction (see Table 4) revealed that RT2 of the auditory-visual task was longer than that of any other modalities; RT2 of the tactile-auditory task was longer than that of visual-auditory, tactile-visual, and visual-tactile tasks; RT2 of the auditory-visual task was longer than that of the visualauditory task; differences in other comparisons were insignificant. The difference in RT2 between modalities could be partly ascribed to the task difficulty: the auditory task was the most difficult, followed by the tactile task, and the visual task was the least difficult (see the next section for details).

Regarding the interaction, the difference in RT2 between modalities (mostly between the auditory-tactile task and the others) gradually became smaller as the SOA increased (see Fig. 3). At a short SOA, the features of Target 1 have more influence on RT2; at s longer SOA, the features of Target 2 have more influence on RT2. We noticed that the slope of the auditory-tactile task seems steeper than the other conditions. Results of a one-sample t-test showed that the slope of RT2 of the auditory-tactile task with SOA less than $1,000 \mathrm{~ms}(M=$ -1.22 , CI $95 \%=[-1.05,-1.39])$ was less than $-1, t(29)=-$ $2.599, p=.015$. This is predicted only by the executive control of theory of visual attention.

The effect of SOA, modalities, and their interaction were all significant on accuracy of Task 2 . Further analysis revealed that the accuracy of Task 2 was affected by SOA only in the auditory-tactile condition (see Table 3): accuracy generally increased from $83 \%$ to $95 \%$ as the SOA increased (see Table 1). In other modalities, accuracy stayed above $95 \%$. This was very similar to the attentional blink effect, which will be discussed later.

We carried out additional analysis to explore whether the relative worse performance in auditory-tactile condition (accuracy of Task 2 depending on SOA and RT2 of a slope less than -1 ) was caused by the forward crosstalk effect. Table 9 presents the main method and results. Neither RT nor accuracy of Task 2 was affected by the congruence between two tasks or its interaction with SOA. The relatively worse performance in the auditory-tactile condition could not be attributed to the forward crosstalk effect.

\section{Performance of Task 1}

Tables 5, 6, 7 in the appendix present the descriptive statistics and results of ANOVA of Task 1 performance. As Table 7 illustrates, the effect of SOA on RT1 was significant in the tactile-visual and auditory-tactile conditions: RT1 generally decreased as the SOA increased. Accuracy of task 1 was not affected by SOA in any modality and stayed above $94 \%$.

To explore whether the task difficulty differs between modalities, we conducted a repeated-measure ANOVA of S1 modality's effect on Task 1 performance, see Table 8 . Accuracy of the visual task was larger than the auditory and the tactile tasks, but the latter two did not differ from each other $(p=.141)$. RT of the visual task was shorter than the auditory and tactile tasks, and RT of the tactile task shorter than that of the auditory task. Therefore, the auditory task was the most difficult, the visual task was the easiest, and the tactile task came somewhere in between.

We carried out additional analysis to explore whether the effect of SOA on RT1 in the auditory-tactile and tactile-visual conditions was caused by backward crosstalk effect. Table 9 presents the main method and results. In the auditory-tactile condition, the effect of congruency on accuracy of Task 1 and the interaction between congruence and SOA on RT and accuracy of Task 1 were significant. No significant effect was found in the tactile-visual condition. In other words, we observed backward crosstalk effect only in the auditory-tactile but not the tactile-visual condition.

\section{Discussion}

In this study, PRP, i.e., the effect of SOA on RT2, exists in all the cross-modal combinations of vision, audition, and tactility. This is consistent with previous studies (Brebner, 1977; Hibberd et al., 2013). Only the executive control of the theory of visual attention could predict all the results in the auditorytactile condition: the RT1 increased, and the accuracy of Task 2 decreased, with a decreasing SOA; the slope of RT2 against SOA was less than -1 ; and a significant backward crosstalk effect was observed. In the tactile-visual condition, the RT1 increased with a decreasing SOA, which could be explained by the central capacity-sharing model. Results of the other combinations of modalities could be explained by both RSB and the central capacity-sharing models.

The result of accuracy of Task 2 in the auditory-tactile condition resembles the attentional blink effect: accuracy of Task 2 decreases as the SOA between two stimuli decreases (Raymond, Shapiro, \& Arnell, 1992). The PRP and attentional blink effects have been found at the same time when RT1 is extremely long (Marti, Sigman, \& Dehaene, 2012). The typical attentional blink paradigm would employ rapid serial visual presentation and back-masking for the second target (Giesbrecht \& Di Lollo, 1998; but see Jannati, Spalek, \& Lollo, 2011). In this study, however, we found an attentional blink effect in the auditory-tactile condition with only two stimuli and no back-masking. This could be partly explained by the higher difficulty and thus longer RT of the auditory task (see Table 8), which led to the loss of the information of S2 in 
Table 1 Mean $(S D)$ RT and accuracy of Task 2

\begin{tabular}{|c|c|c|c|c|c|c|}
\hline \multirow[t]{2}{*}{$\mathrm{SOA}(\mathrm{ms})$} & \multicolumn{6}{|l|}{ Modality } \\
\hline & AT & AV & TV & TA & VA & VT \\
\hline \multicolumn{7}{|l|}{ RT } \\
\hline 75 & $1.54(0.26)$ & $1.25(0.24)$ & $1.11(0.26)$ & $1.27(0.29)$ & $1.07(0.20)$ & $1.12(0.25)$ \\
\hline 150 & $1.49(0.30)$ & $1.19(0.27)$ & $0.99(0.24)$ & $1.23(0.33)$ & $0.98(0.16)$ & $1.04(0.27)$ \\
\hline 300 & $1.23(0.28)$ & $1.00(0.25)$ & $0.80(0.19)$ & $1.07(0.21)$ & $0.90(0.16)$ & $0.86(0.23)$ \\
\hline 600 & $0.90(0.25)$ & $0.77(0.24)$ & $0.67(0.14)$ & $0.97(0.20)$ & $0.81(0.16)$ & $0.76(0.25)$ \\
\hline 1200 & $0.79(0.20)$ & $0.66(0.18)$ & $0.59(0.11)$ & $0.79(0.18)$ & $0.76(0.14)$ & $0.67(0.22)$ \\
\hline \multicolumn{7}{|l|}{ Accuracy } \\
\hline 75 & $.83(.17)$ & $1.00(.02)$ & $.99(.03)$ & $.96(.11)$ & $.98(.05)$ & $.96(.07)$ \\
\hline 150 & $.88(.10)$ & $.98(.04)$ & $1.00(.03)$ & $.96(.07)$ & $.99(.03)$ & $.96(.07)$ \\
\hline 300 & $.92(.09)$ & $.99(.02)$ & $1.00(.00)$ & $.97(.06)$ & $.98(.05)$ & $.97(.07)$ \\
\hline 600 & $.95(.08)$ & $.98(.04)$ & $.98(.05)$ & $.96(.06)$ & $.97(.06)$ & $.98(.04)$ \\
\hline 1200 & $.94(.08)$ & $.98(.05)$ & $1.00(.02)$ & $.98(.05)$ & $.98(.05)$ & $.95(.11)$ \\
\hline
\end{tabular}

Note. SOA stimuli onset asynchrony, $R T$ reaction time, measured in seconds, $A T$ auditory-tactile, $A V$ auditory-visual, $T A$ tactile-auditory, $T V$ tactile-visual, $V A$ visual-auditory, $V T$ visual-tactile

working memory. Furthermore, to explain the difference between auditory-tactile and auditory-visual conditions we need to refer to some modality features: Visual information can linger for a while after the stimuli disappear, in the form of an after-image and visual-spatial scratchpad; however, there is no such counterpart for tactile stimuli, which might be more easily lost. In another study (Rau, Zheng, Wang, Zhao, \& Wang, in press), we found that performing auditory-tactile tasks generally resulted in higher accuracy than performing pure tactile tasks. Combining results from the two studies, we speculated that the accuracy of performing dual-task will be highest if the tasks were distributed across visual and (then) tactile modality, followed by auditory-tactile tasks, and pure tactile tasks came in the last.

In both auditory-tactile and tactile-visual conditions, RT1 also decreases as SOA increases. This violated the prediction of the RSB models, but could be explained by both the central capacity-sharing model and the executive control of the theory of visual attention: a relatively small portion of the central capacity had been allocated to Task 2 when SOA is short, so less resource was allocated to Task 1 and RT1 became longer. This was further supported by the backward crosstalk effect in the auditory-tactile (but not the tactile-visual) condition: early processing of response selection of Task 2 affected the response selection of Task 1. To explain the difference between auditory-tactile and tactile-visual conditions we need to refer to modality features again: Somatosensory processing relies on (the postcentral gyrus of) the right hemisphere more than the left one (Coghill et al., 2001; Symonds et al., 2006; Vallar, 2007). The response execution of Task 1 , which is performed with the left hand, was controlled by the precentral gyrus in the right hemisphere. There was a proximity in cerebral cortex between $\mathrm{R} 1$ and processing of a tactile Task 2. Another explanation is the similarity between auditory and tactile stimuli. Auditory stimuli, i.e., sound waves, are physical vibration. Vibrations

Table 2 Effect of modality, SOA, and their interaction on RT and accuracy of Task 2

\begin{tabular}{|c|c|c|c|c|c|}
\hline Factor & $F$ & $d f$ & $p$ & $\eta_{p}^{2}$ & $1-\beta$ \\
\hline \multicolumn{6}{|l|}{ on RT } \\
\hline Modality & 31.44 & $3.73,108.31$ & $<.001$ & .520 & $>.999$ \\
\hline SOA & 337.46 & $2.41,69.89$ & $<.001$ & .921 & $>.999$ \\
\hline $\begin{array}{l}\text { Modality } * \text { SOA } \\
\text { on accuracy }\end{array}$ & 10.78 & $9.61,278.59$ & $<.001$ & .271 & $>.999$ \\
\hline Modality & 30.674 & $2.99,86.80$ & $<.001$ & .514 & $>.999$ \\
\hline SOA & 3.435 & 4,116 & .011 & .106 & .843 \\
\hline Modality * SOA & 3.348 & $6.55,190.05$ & .003 & .104 & .948 \\
\hline
\end{tabular}

Note. $R T$ reaction time, SOA stimuli onset asynchrony 


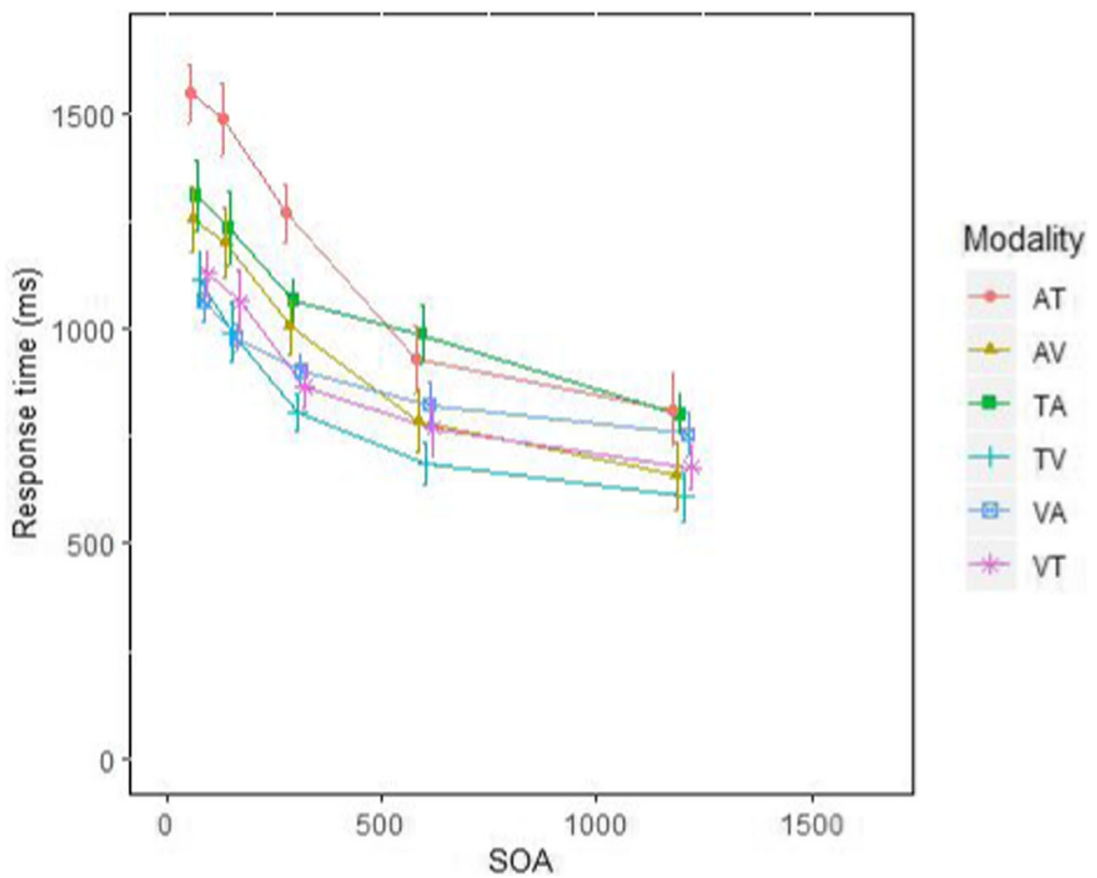

Fig. 3. Response time of Task 2 on stimuli onset asynchrony (SOA, measured in ms) by modalities. Error bars represent CI $95 \%$. $A T$ auditory-tactile, $A V$ auditory-visual, $T A$ tactile-auditory, $T V$ tactile-visual, $V A$ visual-auditory, $V T$ visual-tactile

inevitably emit noise (that is why noise-cancelling earphones were used).

As mentioned above, in the auditory-tactile condition, the slope of RT2 against SOA was less than -1 , which was predicted by the executive control of the theory of visual attention only: the early processing of Task 2 was largely lost and needed to start from almost zero after the response selection of Task 1. Neither the RSB nor the central capacity-sharing models could explain this result.

\section{Limitations and directions for future studies}

The responses in this study, i.e., moving and then clicking, were more complex than the key-pressing used in most RT experiments. The RT included both the time for moving from home position to the target and the time for clicking the trigger. This will generally make the RT longer. Besides, performing complex manual responses with both hands may lead to bimanual interference (Scalf, Banich, Kramer,

Table 3 Effect of SOA on RT and accuracy of Task 2 in different modalities

\begin{tabular}{|c|c|c|c|c|c|}
\hline Modality & $F$ & $d f$ & $p$ & $\eta_{p}^{2}$ & $1-\beta$ \\
\hline \multicolumn{6}{|l|}{$\mathrm{RT}$} \\
\hline VA & 50.92 & $3.04,88.23$ & $<.001$ & .637 & $>.999$ \\
\hline AV & 104.71 & $2.64,76.56$ & $<.001$ & .783 & $>.999$ \\
\hline $\mathrm{VT}$ & 100.84 & $2.78,80.63$ & $<.001$ & .777 & $>.999$ \\
\hline TV & 91.11 & 4,116 & $<.001$ & .759 & $>.999$ \\
\hline AT & 111.93 & 4,116 & $<.001$ & .794 & $>.999$ \\
\hline $\mathrm{TA}$ & 57.69 & $2.98,86.55$ & $<.001$ & .665 & $>.999$ \\
\hline \multicolumn{6}{|l|}{ Accuracy } \\
\hline VA & 0.886 & $2.97,86.11$ & .451 & .030 & .235 \\
\hline AV & 1.129 & $2.66,77.27$ & .339 & .037 & .277 \\
\hline $\mathrm{VT}$ & 0.762 & $2.89,83.88$ & .514 & .026 & .204 \\
\hline TV & 0.508 & $2.87,83.34$ & .670 & .017 & .147 \\
\hline AT & 6.757 & $2.58,74.96$ & .001 & .189 & .952 \\
\hline TA & 2.148 & $2.46,71.21$ & .113 & .069 & .474 \\
\hline
\end{tabular}

Note. $\mathrm{p}<.0083$ indicated significance as corrected with Bonferroni 
Table 4 Difference in RT2 (ms) between modalities with Bonferroni correction

\begin{tabular}{llllll}
\hline Modality & AT & TA & AV & VA & TV \\
\hline TA & $235^{*}$ & & & & \\
AV & $290^{*}$ & $-55^{*}$ & & & \\
VA & $479^{*}$ & $244^{*}$ & $189^{*}$ & & \\
TV & $429^{*}$ & $194^{*}$ & $139^{*}$ & $50^{*}$ & \\
VT & $417^{*}$ & $181^{*}$ & $127^{*}$ & $-62^{*}$ & $-12^{*}$ \\
\hline
\end{tabular}

Note. $A T$ auditory-tactile, $A V$ auditory-visual, $T A$ tactile-auditory, $T V$ tactile-visual, $V A$ visual-auditory, $V T$ visual-tactile

${ }^{*} p<.05$

Narechania, \& Simon, 2007; but see Swinnen \& Wenderoth, 2004), and the interference might also affect the RT. However, the bimanual interference is not moderated by SOA (Huestegge \& Koch, 2010), and it should be independent of stimulus modality. Moreover, as suggested by Fig. 3, the issue of RT measurement did not obscure the PRP effect. Still, we recommend future studies use simple responses such as key pressing.

The two tasks in this study both required semantic processing, and the dual-task costs may arise from the interference (Wickens, 2002; Wu \& Liu, 2008). Future studies could consider adding the task types as a factor (Wahn \& König, 2017). PRP effect becomes weaker when two responses are of different modalities (Klapp et al., 2019). Future studies should explore the cross-modal PRP with better control of inputoutput modality pairing (Hazeltine et al., 2006), using vocal responses (Hazeltine \& Ruthruff, 2006) and even eye movements (Pashler, 1993) besides manual responses, though they also need to consider the RT issue mentioned above.

Comparison of RT2 between the visual-tactile and visualauditory conditions and between the auditory-tactile and auditory-visual conditions did not support the assumed compatibility between haptic input and manual output. Whether there is a compatible output modality for haptic input is left for future studies to explore. There are still possible ideomotor compatibilities within this pairing, i.e., pressing the touchscreen harder when a strong vibration is felt. This could be easily tested with smart phones nowadays (e.g., iPhone X). The task difficulty between modalities was not matched in this study, and this might make the results more complicated. Future multisensory studies should consider matching the task difficulty before the experiments (see Riggs \& Sarter, 2019).

PRP exists in all the modality combinations: The results in the auditory-tactile condition are best explained by the executive control of the theory of visual attention; the results in the tactile-visual condition was best explained by the central capacity-sharing model; the results of other conditions could be explained by both the RSB and the central capacity-sharing models. Processing of visual, auditory, and tactile information are all limited by the central capacity. However, the difference

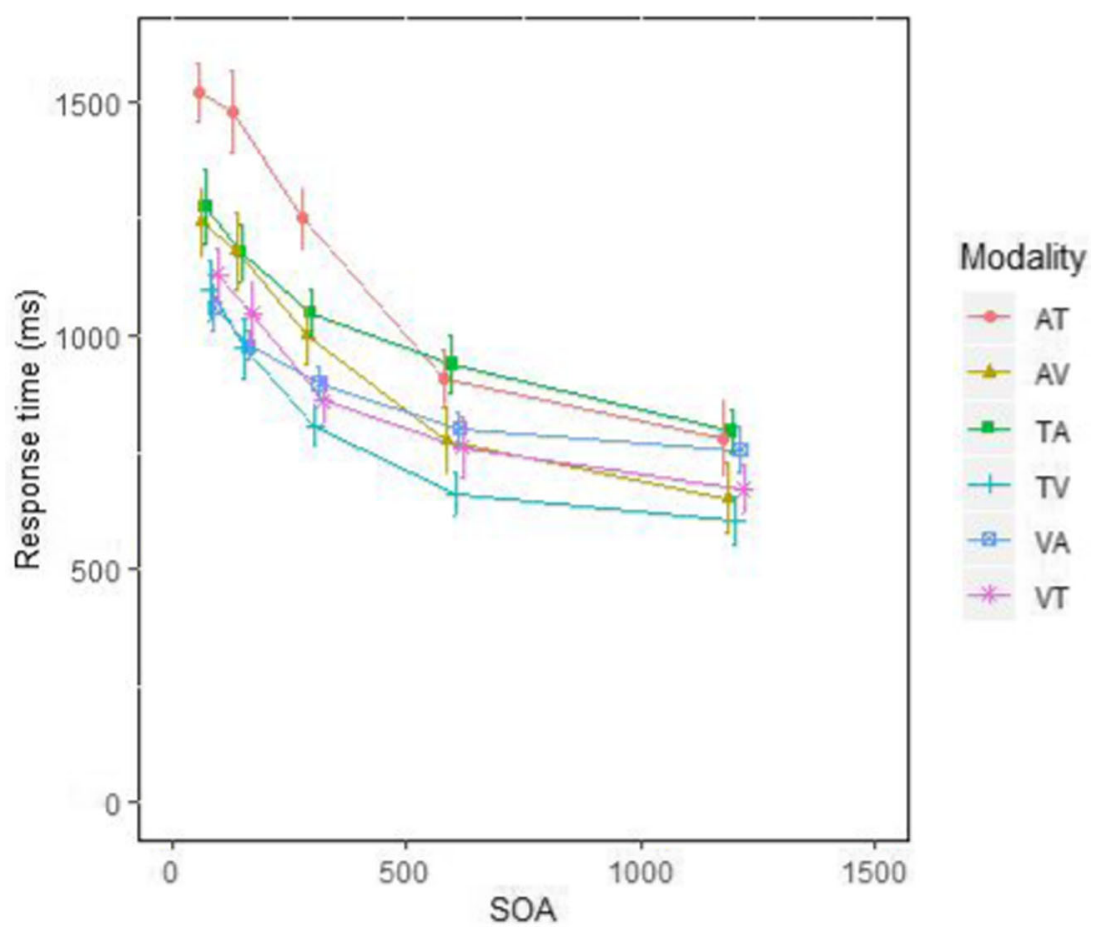

Fig. 4. Response time of Task 2 on stimuli onset asynchrony (SOA, measured in $\mathrm{ms}$ ) by modalities, after excluding trials with incorrect responses. Error bars represent $95 \%$ confidence intervals. AT auditorytactile, $A V$ auditory-visual, $T A$ tactile-auditory, $T V$ tactile-visual, $V A$

visual-auditory, $V T$ visual-tactile. Effect of SOA on RT2 was significant in all the modalities, $p$ values $<.001, \eta_{p}^{2} \geq .66,1-\beta>.999$ 
between modalities cannot be explained by any of those modality-independent theories, but could be explained by modality features to some extent. New theories incorporating input (and maybe even output) modalities into the model are needed to account for those (and future) results.
Acknowledgement This study was funded by the National Key Research and Development Plan 2016YFB1001200.

Open Practices Statements The data are available at https:/osf.io/ $\mathrm{k} 94 \mathrm{t} 5 /$, the materials are not available, and the experiment was not preregistered.

\section{Appendix}

Table 5 Means and SDs of response time and accuracy data in Task 1

\begin{tabular}{|c|c|c|c|c|c|c|}
\hline \multirow[t]{2}{*}{ SOA (ms) } & \multicolumn{6}{|l|}{ Modality } \\
\hline & AT & AV & TA & TV & VA & VT \\
\hline \multicolumn{7}{|l|}{ RT1 } \\
\hline 75 & $1.15(0.19)$ & $1.02(0.18)$ & $0.86(0.20)$ & $0.93(0.22)$ & $0.75(0.15)$ & $0.81(0.18)$ \\
\hline 150 & $1.16(0.24)$ & $1.02(0.22)$ & $0.81(0.20)$ & $0.90(0.24)$ & $0.74(0.15)$ & $0.82(0.21)$ \\
\hline 300 & $1.12(0.23)$ & $0.97(0.20)$ & $0.80(0.18)$ & $0.83(0.20)$ & $0.74(0.17)$ & $0.77(0.17)$ \\
\hline 600 & $0.99(0.22)$ & $0.97(0.26)$ & $0.79(0.22)$ & $0.83(0.19)$ & $0.72(0.20)$ & $0.78(0.27)$ \\
\hline 1,200 & $1.01(0.25)$ & $1.01(0.34)$ & $0.85(0.30)$ & $0.83(0.26)$ & $0.73(0.24)$ & $0.82(0.35)$ \\
\hline \multicolumn{7}{|l|}{$\mathrm{ACC} 1$} \\
\hline 75 & $.97(.05)$ & $.97(.06)$ & $.96(.08)$ & $.95(.06)$ & $.99(.03)$ & $.99(.04)$ \\
\hline 150 & $.95(.07)$ & $.96(.06)$ & $.97(.06)$ & $.94(.09)$ & $1.00(.02)$ & $.98(.05)$ \\
\hline 300 & $.98(.05)$ & $.99(.03)$ & $.98(.04)$ & $.97(.07)$ & $.99(.04)$ & $.99(.05)$ \\
\hline 600 & $.98(.05)$ & $.98(.05)$ & $.97(.05)$ & $.96(.06)$ & $.99(.04)$ & $.99(.02)$ \\
\hline 1,200 & $.99(.04)$ & $.99(.03)$ & $.98(.04)$ & $.99(.04)$ & $.99(.03)$ & $.99(.04)$ \\
\hline
\end{tabular}

Note. SOA stimuli onset asynchrony, $R T 1$ reaction time to the first stimulus, measured in seconds, $A C C 1$ accuracy of Task $1, A T$ auditory-tactile, $A V$ auditory-visual, $T A$ tactile-auditory, $T V$ tactile-visual, $V A$ visual-auditory, $V T$ visual-tactile

Table 6 Effect of modality and stimuli onset asynchrony (SOA) on response time and accuracy of Task 1

\begin{tabular}{lrrrr}
\hline Source & $F$ & $d f$ & $p$ & $\eta_{p}^{2}$ \\
\hline on RT1 & & & & \\
Modality & 33.522 & $3.62,104.88$ & $<.001$ & .536 \\
SOA & 6.227 & $1.86,53.99$ & .004 & .177 \\
Modality * SOA & 3.101 & $8.67,251.57$ & .002 & .097 \\
on ACC1 & & & .969 \\
Modality & 5.788 & 5,145 & .001 & .166 \\
SOA & 5.184 & 4,116 & .001 & .152 \\
Modality * SOA & 0.928 & $10.26,297.42$ & .509 & .031 \\
\hline
\end{tabular}

Note. $R T 1$ reaction time to the first stimulus, $A C C 1$ accuracy of Task 1 
Table 7 Effects of stimuli onset asynchrony (SOA) on response time and accuracy of Task 1 in different modalities

\begin{tabular}{|c|c|c|c|c|c|}
\hline Modality & $F$ & $d f$ & $p$ & $\eta_{p}^{2}$ & $1-\beta$ \\
\hline \multicolumn{6}{|l|}{ RT1 } \\
\hline VA & 0.531 & $2.90,84.09$ & .656 & .018 & .153 \\
\hline AV & 1.062 & $2.05,59.54$ & .354 & .035 & .230 \\
\hline VT & 1.053 & $1.98,57.44$ & .355 & .035 & .225 \\
\hline TV & 4.777 & 4,116 & .001 & .141 & .947 \\
\hline AT & 9.594 & 4,116 & $<.001$ & .249 & $>.999$ \\
\hline TA & 3.158 & $2.90,84.23$ & .030 & .098 & .704 \\
\hline \multicolumn{6}{|l|}{$\mathrm{ACC} 1$} \\
\hline VA & 0.595 & $3.04,88.04$ & .622 & .020 & .170 \\
\hline $\mathrm{AV}$ & 2.083 & $3.15,91.38$ & .105 & .067 & .531 \\
\hline VT & 0.534 & $2.86,82.89$ & .652 & .018 & .152 \\
\hline TV & 2.060 & 4,116 & .090 & .066 & .599 \\
\hline AT & 1.900 & $2.92,84.64$ & .137 & .061 & .469 \\
\hline TA & 1.028 & $3.11,90.18$ & .386 & .034 & .276 \\
\hline
\end{tabular}

Note. $p<.0083$ indicates significance as corrected with Bonferroni

Significant effects aree shown in bold

$R T 1$ reaction time to the first stimulus, measured in seconds, $A C C 1$ accuracy of Task 1, $A C C 2$ accuracy of Task 2, AT auditory-tactile, $A V$ auditory-visual, $T A$ tactile-auditory, $T V$ tactile-visual, $V A$ visual-auditory, $V T$ visual-tactile

Table 8 Mean $(S D)$ of accuracy and response time of Task 1 and the effect of modality

\begin{tabular}{|c|c|c|c|c|c|c|c|}
\hline Variable & Visual & Auditory & Tactile & $F(2,58)$ & $p$ & $\eta_{p}^{2}$ & $1-\beta$ \\
\hline Accuracy & $.991(.012)$ & $.976(.019)$ & $.968(.021)$ & 72.531 & $<.001$ & .714 & $>.999$ \\
\hline Response time & $0.76(0.18)$ & $1.04(0.17)$ & $0.85(0.19)$ & 16.223 & $<.001$ & .359 & .999 \\
\hline
\end{tabular}


Table 9 Effects of stimuli onset asynchrony (SOA) and congruency on task performance in AT and TV conditions

\begin{tabular}{|c|c|c|c|c|c|}
\hline Modality & Source & $\mathrm{F}(1,29)$ & $p$ & $\eta_{p}^{2}$ & $1-\beta$ \\
\hline \multirow[t]{16}{*}{ AT } & on RT1 & & & & \\
\hline & SOA & 15.984 & $<.001$ & .355 & .971 \\
\hline & Congruency & 0.204 & .655 & .007 & .072 \\
\hline & SOA * Congruency & 13.307 & .001 & .315 & .941 \\
\hline & on $\mathrm{ACC} 1$ & & & & \\
\hline & SOA & 5.431 & .027 & .158 & .615 \\
\hline & Congruency & 4.196 & .050 & .126 & .508 \\
\hline & SOA * Congruency & 9.177 & .005 & .240 & .833 \\
\hline & on RT2 & & & & \\
\hline & SOA & 104.529 & $<.001$ & .783 & $>.999$ \\
\hline & Congruency & 1.792 & .191 & .058 & .253 \\
\hline & SOA * Congruency & 2.447 & .129 & .078 & .328 \\
\hline & on $\mathrm{ACC} 2$ & & & & \\
\hline & SOA & 10.458 & .003 & .265 & .878 \\
\hline & Congruency & 0.085 & .773 & .003 & .059 \\
\hline & SOA * Congruency & 0.035 & .853 & .001 & .054 \\
\hline \multirow[t]{8}{*}{ TV } & on RT1 & & & & \\
\hline & SOA & 1.354 & .254 & .045 & .203 \\
\hline & Congruency & 3.138 & .087 & .098 & .402 \\
\hline & SOA * Congruency & 1.370 & .251 & .045 & .205 \\
\hline & on ACC1 & & & & \\
\hline & SOA & 2.294 & .141 & .073 & .311 \\
\hline & Congruency & 0.103 & .750 & .004 & .061 \\
\hline & SOA * Congruency & 1.303 & .263 & .043 & .197 \\
\hline
\end{tabular}

Note. $R T 1$ reaction time to the first stimulus, $R T 2$ reaction time to the second stimulus, $A C C 1$ accuracy of Task 1, ACC2 accuracy of Task $2, S O A$ stimuli onset asynchrony, $A T$ auditory-tactile, $T V$ tactile-visual

Significant effects are shown in bold. Here SOA had only two levels: short (75 or $150 \mathrm{~ms}$ ) and long (600 or 1,200 ms). Congruency denotes whether the responses of the two tasks were the same (congruent, both small or both large) or different (incongruent)

\section{References}

Arnell, K. M., \& Larson, J. M. (2002). Cross-modality attentional blinks without preparatory task-set switching. Psychonomic Bulletin \& Review, 9(3), 497-506.

Brebner, J. (1977). The search for exceptions to the psychological refractory period. Attention and Performance VI, 63-78.

Bundesen, C. (1990). A theory of visual attention. Psychological Review, 97(4), 523-547. https://doi.org/https://doi.org/10.1037/0033-295x. 97.4.523

Chun, M. M., \& Potter, M. C. (1995). A two-stage model for multiple target detection in rapid serial visual presentation. Journal of Experimental Psychology. Human Perception and Performance, 21(1), 109-127.

Coghill, R. C., Gilron, I., \& Iadarola, M. J. (2001). Hemispheric lateralization of somatosensory processing. Journal of Neurophysiology,
85(6), 2602-2612. https://doi.org/https://doi.org/10.1152/jn.2001. 85.6.2602

Collignon, O., \& De Volder, A. G. (2009). Further evidence that congenitally blind participants react faster to auditory and tactile spatial targets. Canadian Journal of Experimental Psychology, 63(4), 287-293. https://doi.org/https://doi.org/10.1037/a0015415

Dux, P. E., Ivanoff, J., Asplund, C. L., \& Marois, R. (2006). Isolation of a central bottleneck of information processing with time-resolved FMRI. Neuron, 52(6), 1109-1120. https://doi.org/https://doi.org/ 10.1016/j.neuron.2006.11.009

Faul, F., Erdfelder, E., Lang, A.-G., \& Buchner, A. (2007). G* Power 3: A flexible statistical power analysis program for the social, behavioral, and biomedical sciences. Behavior Research Methods, 39, 175-191.

Giesbrecht, B., \& Di Lollo, V. (1998). Beyond the attentional blink: Visual masking by object substitution. Journal of Experimental Psychology. Human Perception and Performance, 24(5), 1454 1466. 
Greenwald, A. G. (1972). On doing two things at once: Time sharing as a function of ideomotor compatibility. Journal of Experimental Psychology, 94(1), 52-57.

Greenwald, A. G., \& Shulman, H. G. (1973). On doing two things at once. II. Elimination of the psychological refractory period effect. Journal of Experimental Psychology, 101(1), 70-76.

Hazeltine, E., \& Ruthruff, E. (2006). Modality pairing effects and the response selection bottleneck. Psychological Research, 70(6), 504-513. https://doi.org/https://doi.org/10.1007/s00426-005-0017-

Hazeltine, E., Ruthruff, E., \& Remington, R. W. (2006). The role of input and output modality pairings in dual-task performance: Evidence for content-dependent central interference. Cognitive Psychology, 52(4), 291-345. https://doi.org/https://doi.org/10.1016/j.cogpsych. 2005.11.001

Hibberd, D. L., Jamson, S. L., \& Carsten, O. M. J. (2013). Mitigating the effects of in-vehicle distractions through use of the Psychological Refractory Period paradigm. Accident Analysis \& Prevention, 50, 1096-1103. https://doi.org/https://doi.org/10.1016/j.aap.2012.08. 016

Hommel, B. (1998). Automatic stimulus-response translation in dual-task performance. Journal of Experimental Psychology. Human Perception and Performance, 24(5), 1368-1384. https://doi.org/ https://doi.org/10.1037//0096-1523.24.5.1368

Hommel, B., \& Eglau, B. (2002). Control of stimulus-response translation in dual-task performance. Psychological Research, 66(4), 260 273. https://doi.org/https://doi.org/10.1007/s00426-002-0100-y

Huestegge, L., \& Hazeltine, E. (2011). Crossmodal action: Modality matters. Psychological Research, 75(6), 445-451. https://doi.org/ https://doi.org/10.1007/s00426-011-0373-0

Huestegge, L., \& Koch, I. (2010). Crossmodal action selection: Evidence from dual-task compatibility. Memory \& Cognition, 38(4), 493-501. https://doi.org/https://doi.org/10.3758/MC.38.4.493

Hunt, A. R., \& Kingstone, A. (2004). Multisensory executive functioning. Brain and Cognition, 55(2), 325-327. https://doi.org/https:// doi.org/10.1016/j.bandc.2004.02.072

Janczyk, M., Renas, S., \& Durst, M. (2018). Identifying the locus of compatibility-based backward crosstalk: Evidence from an extended PRP paradigm. Journal of Experimental Psychology: Human Perception and Performance, 44(2), 261-276. http://dx.doi.org/ https://doi.org/10.1037/xhp0000445

Jannati, A., Spalek, T. M., \& Lollo, V. D. (2011). Neither backward masking of T2 nor task switching is necessary for the attentional blink. Psychonomic Bulletin \& Review, 18(1), 70-75. https:// doi.org/https://doi.org/10.3758/s13423-010-0015-3

Klapp, S. T., Maslovat, D., \& Jagacinski, R. J. (2019). The bottleneck of the psychological refractory period effect involves timing of response initiation rather than response selection. Psychonomic Bulletin \& Review, 26(1), 29-47. https://doi.org/https://doi.org/10. 3758/s13423-018-1498-6

Lakens, D. (2013). Calculating and reporting effect sizes to facilitate cumulative science: a practical primer for t-tests and ANOVAs. Frontiers in Psychology, 4, 1-12. https://doi.org/https://doi.org/10. 3389/fpsyg.2013.00863

Lien, M.-C., McCann, R. S., Ruthruff, E., \& Proctor, R. W. (2005). Dualtask performance with ideomotor-compatible tasks: Is the central processing bottleneck intact, bypassed, or shifted in locus? Journal of Experimental Psychology. Human Perception and Performance, 31(1), 122-144. https://doi.org/https://doi.org/10.1037/0096-1523. 31.1 .122
Lien, M.-C., Proctor, R. W., \& Allen, P. A. (2002). Ideomotor compatibility in the psychological refractory period effect: 29 years of oversimplification. Journal of Experimental Psychology. Human Perception and Performance, 28(2), 396-409.

Logan, G. D., \& Gordon, R. D. (2001). Executive control of visual attention in dual-task situations. Psychological Review, 108(2), 393-434. https://doi.org/https://doi.org/10.1037/0033-295x.108.2.393

Marti, S., Sigman, M., \& Dehaene, S. (2012). A shared cortical bottleneck underlying Attentional Blink and Psychological Refractory Period. NeuroImage, 59(3), 2883-2898. https://doi.org/https://doi.org/10. 1016/j.neuroimage.2011.09.063

Morrison, N. M. V., Burnham, D., \& Morrison, B. W. (2015). Cognitive Load in Cross-Modal Dual-Task Processing. Applied Cognitive Psychology, 29(3), 436-444. https://doi.org/https://doi.org/10. 1002/acp.3122

Nosofsky, R. M., \& Palmeri, T. J. (1997). An exemplar-based random walk model of speeded classification. Psychological Review, 104(2), 266-300. https://doi.org/https://doi.org/10.1037/0033-295x.104.2. 266

Pashler, H. (1994). Dual-task interference in simple tasks: data and theory. Psychological Bulletin, 116, 220-244.

Pashler, H., Carrier, M., \& Hoffman, J. (1993). Saccadic eye movements and dual-task interference. The Quarterly Journal of Experimental Psychology. A, Human Experimental Psychology, 46, 46(1), 51-82.

Rau, P.-L. P., \& Zheng, J. (2019). Crossmodal PRP. Retrieved February 18,2019 , from osf.io/k94t5

Rau, P.-L. P., Zheng, J., Wang, L., Zhao, J., \& Wang, D. (in press). Haptic and Auditory-haptic Attentional Blink in Spatial and Object-based Tasks. Multisensory Research.

Raymond, J. E., Shapiro, K. L., \& Arnell, K. M. (1992). Temporary suppression of visual processing in an RSVP task: An attentional blink? Journal of Experimental Psychology. Human Perception and Performance, 18(3), 849-860.

Riggs, S. L., \& Sarter, N. (2019). Crossmodal Matching: The Case for Developing and Employing a Valid and Feasible Approach to Equate Perceived Stimulus Intensities in Multimodal Research. Human Factors, 61(1), 29-31. https://doi.org/https://doi.org/10. $1177 / 0018720818816439$

Ruthruff, E., Pashler, H., \& Hazeltine, E. (2003). Dual-task interference with equal task emphasis: Graded capacity sharing or central postponement? Perception \& Psychophysics, 65(5), 801-816. https:// doi.org/10.3758/BF03194816

Ruthruff, E., Johnston, J. C., \& Remington, R. W. (2009). How strategic is the central bottleneck: Can it be overcome by trying harder? Journal of Experimental Psychology: Human Perception and Performance, 35(5), 1368-1384. https://doi.org/10.1037/a0015784.

Scalf, P. E., Banich, M. T., Kramer, A. F., Narechania, K., \& Simon, C. D. (2007). Double take: Parallel processing by the cerebral hemispheres reduces attentional blink. Journal of Experimental Psychology: Human Perception and Performance, 33(2), 298329. https://doi.org/https://doi.org/10.1037/0096-1523.33.2.298

Sigman, M., \& Dehaene, S. (2008). Brain mechanisms of serial and parallel processing during dual-task performance. Journal of Neuroscience, 28(30), 7585-7598. https://doi.org/https://doi.org/ 10.1523/JNEUROSCI.0948-08.2008

Soto-Faraco, S., Spence, C., Fairbank, K., Kingstone, A., Hillstrom, A. P., \& Shapiro, K. (2002). A crossmodal attentional blink between vision and touch. Psychonomic Bulletin \& Review, 9(4), 731-738. https://doi.org/https://doi.org/10.3758/BF03196328

Stephan, D. N., \& Koch, I. (2010). Central cross-talk in task switching: Evidence from manipulating input-output modality compatibility. 
Journal of Experimental Psychology. Learning, Memory, and Cognition, 36(4), 1075-1081. https://doi.org/10.1037/a0019695.

Strobach, T., Schütz, A., \& Schubert, T. (2015). On the importance of Task 1 and error performance measures in PRP dual-task studies. Frontiers in Psychology, 6, 1-8. https://doi.org/10.3389/fpsyg.2015. 00403

Swinnen, S. P., \& Wenderoth, N. (2004). Two hands, one brain: Cognitive neuroscience of bimanual skill. Trends in Cognitive Sciences, 8(1), $18-25$.

Symonds, L. L., Gordon, N. S., Bixby, J. C., \& Mande, M. M. (2006). Right-Lateralized Pain Processing in the Human Cortex: An fMRI Study. Journal of Neurophysiology, 95(6), 3823-3830. https:// doi.org/https://doi.org/10.1152/jn.01162.2005

Tombu, M., \& Jolicoeur, P. (2003). A central capacity-sharing model of dual-task performance. Journal of Experimental Psychology. Human Perception and Performance, 29(1), 3-18. https://doi.org/ https://doi.org/10.1037//0096-1523.29.1.3
Vallar, G. (2007). A hemispheric asymmetry in somatosensory processing. Behavioral and Brain Sciences, 30(2), 223-224. https://doi.org/ https://doi.org/10.1017/S0140525X0700163X

Wahn, B., \& König, P. (2017). Is Attentional Resource Allocation Across Sensory Modalities Task-Dependent? Advances in Cognitive Psychology, 13(1), 83-96. https://doi.org/https://doi.org/10.5709/ acp-0209-2

Welford, A. T. (1952). The 'psychological refractory period'and the timing of high-speed performance - a review and a theory. British Journal of Psychology. General Section, 43(1), 2-19.

Wickens, C. D. (2002). Multiple resources and performance prediction. Theoretical Issues in Ergonomics Science, 3, 159-177.

Wu, C., \& Liu, Y. (2008). Queuing network modeling of the psychological refractory period (PRP). Psychological Review, 115, 913.

Publisher's note Springer Nature remains neutral with regard to jurisdictional claims in published maps and institutional affiliations. 